\title{
Trans-Aortic or Pulmonary Artery-Based Drainage for Left Ventricular Unloading and Veno-Arterial Extracorporeal Life Support Management in a Porcine Cardiogenic Shock Model.
}

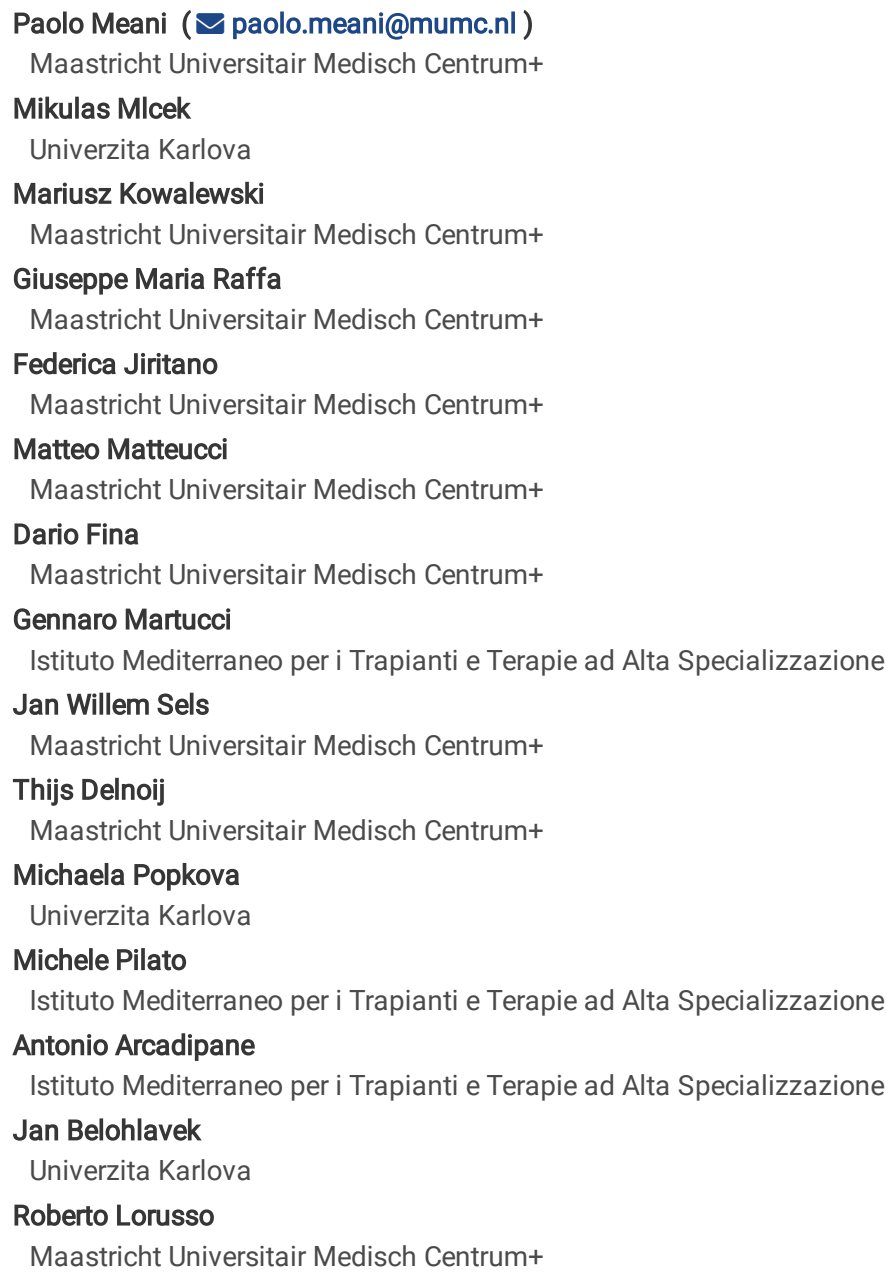

Research

Keywords: ECLS, Cardiogenic Shock, LV Unloading, Impella, Pulmunary Drainage, LV venting, CS Porcine Model

Posted Date: March 4th, 2020

DOI: https://doi.org/10.21203/rs.3.rs-16019/v1

License: @) (7) This work is licensed under a Creative Commons Attribution 4.0 International License. Read Full License

Version of Record: A version of this preprint was published at Seminars in Thoracic and Cardiovascular Surgery on November 1st, 2020. See the published version at https://doi.org/10.1053/j.semtcvs.2020.11.001. 


\section{Abstract}

Background The use of peripheral veno-arterial extracorporeal life support (V-A ECLS) as a mechanical circulatory support in cardiogenic shock has increased dramatically over the last years. However, increased afterload may jeopardize left ventricle (LV) recovery and cause blood stasis and pulmonary edema. Therefore, several LV unloading techniques have been developed and used with limited understanding of the actual difference among them. The aim of the present study was to compare a trans-aortic suction device (Impella) and pulmonary artery (PA) drainage, for LV unloading and V-A ECLS management as well as efficacy in a porcine cardiogenic shock (CS) model

Methods A dedicated CS model compared included twelve female swine (21 \pm 1,8-weeks old and weighing 54,3 $\pm 4,6 \mathrm{~kg})$ supported with V-A ECLS and randomized to Impella or PA-related LV drainage. LV unloading and end-organ perfusion were evaluated through the pulmonary artery catheter and the LV pressure/volume analysis. All the variables were collected at baseline, profound CS, V-A ECLS support with maximum flow and when Impella or PA cannula run on top.

Results CS was successfully induced in all twelve animals. Impella resulted in a marked drop of LVEDV compared to a slight decrease in the PA cannula group, resulting in an overall stroke work (SW) and Pressure-Volume Area (PVA) reductions with both techniques. However, SW reduction was significant in the Impella CP group (VA ECMO $3998.8 \otimes 2027.6 \mathrm{mmHg} \times \mathrm{mL}$ vs VAECMO + Impella $1796.9 \pm 1033.9 \mathrm{mmHg} \times \mathrm{ml}$, $\mathrm{p}$ value 0,016 ), leading to a more consistent PVA reduction (Impella reduction 34,7\% vs PA cannula reduction 9,7\%) In terms of end organ perfusion, central and mixed 02 saturation improved with V-A ECLS, and subsequently, remaining unchanged with either Impella or PA cannula as unloading strategy

Conclusions Trans-aortic suction and PA drainage provided effective LV unloading during V-A ECLS while maintaining adequate end-organ perfusion. Transaortic suction device provides a greater LV unloading effect and reduces more effectively the total LV stroke work.

\section{Background}

The use of mechanical circulatory support (MCS) has increased dramatically over the last 15 years. However, mortality in cardiogenic shock (CS) did not change in the same period, remaining at approximately $50 \%$ in affected patients ${ }^{12}$. One of the most frequently applied MCS in this setting is veno-arterial extracorporeal life support (V-A ECLS) ${ }^{3}$. Despite marked advances in this technology, several complications and inherent shortcomings may reduce the potential benefits. Among these side effects, increased afterload to the left ventricle (LV) represent a critical threat. This complication appears more frequently in peripheral V-A ECLS and may lead to LV dilatation, increases of LV and atrial (LA) end-diastolic pressures, and pulmonary oedema ${ }^{4}$. This LV and LA maladaptive change jeopardize LV recovery, particularly in the presence of ischemia-induced myocardial impairment. and, moreover, VA ECMO negative hemodynamic effect on LV performance is flow dependent ${ }^{5}$ justifying close LV function monitoring and early intervention ${ }^{6}$. In case of extreme overload and severe LV dysfunction, such a condition may lead to protracted aortic valve closure even during systole, causing blood stasis in the LV, thus increasing the risk of thrombi formation ${ }^{7}$. It is therefore clear that the extent of LV unloading, and aortic valve opening should be continuously monitored to timely alert the attending personnel about such a potential negative impact of V-A ECLS on LV performance and indicate the actual need for less or more aggressive maneuvers to facilitate LV decompression.

Several approaches promoting LV unloading are being used clinically, including pharmacological interventions, adjustment of mechanical ventilation parameters, or catheter-based interventions, including transvenous septostomy. The actual timing, effect on the overall patient outcome, and difference of different type of interventions, however, remain unclear ${ }^{4}$. The use of trans-aortic suction device represents one of the most frequently adopted tool to achieve effective LV unloading ${ }^{8}$. Another promising technique accounts for an indirect LV unloading procedure by draining blood from the pulmonary artery (PA). Both techniques represent appealing tool since may be achieved with a percutaneous approach ${ }^{9}$. Therefore, we designed an experimental study of CS managed by V-A ECLS in which the two techniques were randomly applied to assess the effect on the LV unloading and performance, together with the impact on overall ECLS-related end-organ perfusion.

\section{Methods}

The study was approved by Charles University, First Faculty of Medicine Institutional Animal Care and Use Committee and was performed at the university laboratory in accordance with Act No 246/1992 Coll. on the protection of animals against cruelty that is harmonized with EU directives on the protection of animals used for scientific purposes.

Supplementary Appendix 1 and 2 show the description of anesthesia and instrumentation. Briefly, twelve female swine, approximately $21 \pm 1,8$ weeks old and weighing 54,3 \pm 4,6 kg, were randomized to Impella (Impella CP, Abiomed, Danvers, MA, USA,) or PA cannula (Biomedicus VR 9670-019, Medtronic, Minneapolis, MN, USA) as LV unloading technique. The whole procedures were carried out under total i.v. anesthesia (propofol, midazolam, morphine). Initially, hemodynamic and oximetry monitoring systems, including cardiac pressure-volume catheter, were introduced. Then the V-A ECLS circuit was inserted (femorofemoral percutaneous approach) accompanied by selected venting system (Impella via contra-lateral femoral artery or PA cannula via internal right jugular vein). Cardiogenic shock was successfully induced in all twelve animals by percutaneous coronary artery balloon-occlusion. Stepwise ECLS protocol with and without LV unloading was performed. An incremental fixed ECLS support was applied each step, testing the LV unloading provided by Impella or PA cannula at the same ECMO flow. At the end of the protocol the support was withdrawn resulting in hemodynamic collapse thus confirming the severity of CS.

\section{PRESSURE-VOLUME MEASUREMENTS}

A pressure-volume (PV) admittance catheter 7F VSL (Transonic Systems Inc., Ithaca, USA) was percutaneously inserted through a 7-French sheath in the left carotid artery, advanced retrogradely into the LV, and connected to an ADV 500 PV System. The PV system was connected to a PowerLab 16/35

Page 2/10 
(ADInstruments, Dunedin, New Zealand) and the PV measurements were continuously recorded in LabChart Pro (ADInstruments). The admittance catheter acquired the following data: LV pressure, LV volume, phase, magnitude, while the LabChart Pro provided the calculations of multiple PV parameters: enddiastolic pressure (LVEDP, mmHg), LV end-diastolic volume (LVEDV, $\mathrm{mL}$ ), LV stroke work (SW, mmHg'mL), LV end systolic pressure (LVESP, mmHg), LV endsystolic pressure-volume relationship (ESPVR, representing contractility), the LV isovolumic relaxation constant (Tau, representing ventricular relaxation) and pressure-volume area (PVA, $\left.\mathrm{mmHg}^{\prime} \mathrm{mL}\right)$.

The gold standard for estimating ESPVR and PVA is by preload reduction. However, this was not carried out in the current study for two reasons: first, the unsupported profound CS leading to severely compromised hemodynamics didn't allow any further pre-load reductions; second, preload changes during V-A ECLS support would cause a significant impact on device performance. As a result, the latter may potentially influence device-derived afterload, making it difficult to define clearly how ESPVR and PVA changes would have determined by the heart or by the device.

Therefore, Vo (the theoretical volume when no pressure is generated) was arbitrarily assumed as 0 in each animal and was kept as a constant throughout the study to generate single-beat estimations of ESPVR and PVA (Figure 1) ${ }^{1011}$

\section{EXPERIMENTAL PROTOCOL}

At the beginning of the protocol the animals were randomized into Impella or PA cannula group. Our protocol consisted of three main phases: baseline, cardiogenic shock T1 and T2, each lasting 100 minutes. In each phase, V-A ECLS support was increased in a stepwise manner every 20 minutes in increments of $20 \mathrm{ml} / \mathrm{kg}$.min from minimum of $20 \mathrm{ml} / \mathrm{kg}$.min up to the maximum target of $100 \mathrm{ml} / \mathrm{kg}$.min, if achievable. At each ECLS flow level, during first $10 \mathrm{minutes,}$ ECLS was not unloaded followed by 10 minutes of unloading by PA or Impella according to the randomization. The Impella pump speed was set to P-1 (minimum Impella revolutions per minute) and then maximum P level each step. Differently, the PA cannula was open or closed, leading to an average flow of $1,6 \mathrm{I} / \mathrm{min}$. The step details are included in Appendix 1.

As soon as the first "baseline" step was finished, CS was induced. In brief, the proximal/middle Left anterior descending coronary artery was occluded by a regular percutaneous coronary compliant balloon $(4 \times 20 \mathrm{~mm}$ ). The balloon was kept inflated for at least 45 minutes (maximum 60 minutes). The aim was to achieve a profound CS, defined as cardiac output $<50 \mathrm{ml} / \mathrm{kg}$.min and/or a mixed venous oxygen saturation $\leq 50 \%$. If the animal developed ventricular fibrillation during the induction phase, V-A ECLS flow would have been adapted in order to maintain a mean arterial pressure (MAP) at around $50 \mathrm{mmHg}$. At the end of the CS induction, in case of the presence of sustained ventricular malignant arrhythmia, defibrillation was performed (200J biphasic repeated as necessary) in order to restore sinus rhythm. Circulation was stabilized and ECLS support reduced to $20 \mathrm{ml} / \mathrm{kg}$.min.

Thereafter, T1-phase CS protocol was initiated. In case of T1-phase failure ( parameter instability, catheter malfunctioning, record issues), T2-phase could subsequently be implemented. In case that heart failure was recovering unexpectedly fast, ischemia induction was repeated, and T2-phase CS protocol was run after this second ischemia induction. ECLS pump flow was set at $0,5 \mathrm{~L} / \mathrm{min}$ for 15 minutes and then increased by $20 \%$ each step every 10 minutes until achieving maximum planned ECLS flow. V-A ECLS oxygenator (flow and FiO2) was set in order to keep adequate and steady $\mathrm{PaO} 2$ and $\mathrm{PaCO} 2$ ( $\mathrm{PaO} \mathrm{O}_{2}$ between 120-150 mm and $\mathrm{PaCO}_{2}$ " $40 \mathrm{mmHg}$ ). Norepinephrine was continuously administered whenever MAP dropped below $50 \mathrm{mmHg}$ and was discontinued once MAP increased over $60 \mathrm{mmHg}$.

\section{DATA ACQUISITION}

All parameters were continuously recorded in LabChart Pro (ADInstruments).

Hemodynamic parameters as well as V-A ECLS data and PV relationships (LVEDP and LVEDV, PVA, ESPVR, Tau, SW, LV output) were extracted from continuous data at preset time points: 1. baseline, 2. CS (with minimum ECMO support), 3. CS with maximum V-A ECLS support alone and, 4. CS with maximum support plus Impella or PA cannula unloading. Additionally, post capillary wedge pressure (PCWP) and echocardiogram were acquired at end of each step.

\section{STATISTICAL ANALYSIS}

The primary outcomes were LV performance, mainly defined as PVA, SW and potential energy (PE), and end-organ perfusion, described by the central and mixed $\mathrm{O}_{2}$ saturation.

Data are presented as mean (95\% confidence interval) with error bars in the two figures of LVEDV and LVEDP over time representing standard error of the mean. Given the uncertainty of absolute values for LV volume using the conductance method, in order to better underline the specific

unloading effect, a comparison between LV venting techniques was performed in terms of relative change from the CS state only supported with V-A ECLS. Given the small sample size, the Wilcoxon rank-sum test was used to assess individual variable differences between the Impella or PA cannula and V-A ECLS at different time points. Statistical analyses were performed with SAS/STAT (SAS Institute inc., Cary, North Carolina, USA). A p-level $\leq 0.05$ was considered statistically significant.

\section{Results}

\section{BASELINE CHARACTERISTICS}


There were no differences between Impella and PA cannula groups in the measured variables at baseline or at induced CS state (Table 2). Two animals in the Impella group and five animals in the PA cannula group required norepinephrine (max dosage $1600 \mathrm{mcg} / \mathrm{h}$ ) to maintain a mean arterial pressure (MAP) $>50$ $\mathrm{mmHg}$ after CS was established. Furthermore, one animal in the PA cannula group required dopamine (20 mcg/h) in addition to norepinephrine due to junctional rhythm leading to further hemodynamic instability (Table 1).

V-A ECLS maximum flow did not significantly differ in both groups $(4,0 \pm 0,1 \mathrm{~L} / \mathrm{min}$ and 3,8 $\pm 0,9 \mathrm{~L} / \mathrm{min}$-p-value $>0,05$, in Impella and PA cannula groups, respectively). Complete circulatory collapse with pulseless electrical activity developed within a few minutes after withdrawal of MCS in all animals.

Table 1

Experimental details in each animal.

\begin{tabular}{|c|c|c|c|}
\hline & Support & Max dosage $(\mathrm{mcg} / \mathrm{h})$ & Details \\
\hline \multicolumn{4}{|c|}{ IMpella } \\
\hline 1 & Norepinephrine & 1600 & Suction \\
\hline 2 & Norepinephrine & 50 & \\
\hline 3 & & & Extra-beats, Tricuspid regurgitation \\
\hline \multicolumn{4}{|l|}{4} \\
\hline \multicolumn{4}{|l|}{5} \\
\hline \multicolumn{4}{|l|}{6} \\
\hline \multicolumn{4}{|c|}{ PA cannula } \\
\hline 1 & Norepinephrine & 1 & \\
\hline 2 & Norepinephrine & 200 & \\
\hline \multicolumn{4}{|l|}{3} \\
\hline 4 & Norepinephrine/Dopamine & $50 / 20$ & Hemodynamic unstable Junction Rhythm \\
\hline 5 & Norepinephrine & 500 & \\
\hline 6 & Norepinephrine & 600 & \\
\hline
\end{tabular}

\section{HEMODYNAMICS AND END-ORGAN PERFUSION}

V-A ECLS alone was able to increase significantly MAP during CS in all animals (see Table 2).

Thereafter, the introduction of both LV unloading procedures in association with ECLS was differently able to keep MAP steady: the norepinephrine administration was greater for PA cannula animals (5 over 6 ) than for Impella group (2 over 6).

Mean PAP (mPAP) dramatically dropped by approximately $50 \%$ with PA cannula ( VA ECMO alone mPAP $24,3 \pm 11.2$ mmHg vs VA ECMO + PA cannula mPAP $12,4 \pm 5,0 \mathrm{mmHg}$, p value 0,015 ), whereas no significant decrease was recorded with Impella ( VA ECMO alone mPAP $23,6 \pm 6.4 \mathrm{mmHg}$ vs VA ECMO + Impella mPAP $22,0 \pm 6.9 \mathrm{mmHg}, \mathrm{p}$ value 0,677 ). Both configurations led to a modest decremental trend in PCWP.

Finally, central and mixed $\mathrm{O}_{2}$ saturation, as markers of end-organ perfusion, improved with V-A ECLS, and subsequently remained stable steady in both groups throughout the experiment (Table 2). 
Table 2

Haemodynamics and venous oxygen saturations at different time points.

\begin{tabular}{|c|c|c|c|c|c|c|c|c|c|c|}
\hline & & $\begin{array}{l}\text { Baseline } \\
\text { (mean } \pm S D)\end{array}$ & $\begin{array}{l}\text { p-value } \\
\text { Impella vs PA } \\
\text { cannula }\end{array}$ & $\begin{array}{l}\text { CS } \\
\text { (mean } \pm S D)\end{array}$ & $\begin{array}{l}\text { p-value } \\
\text { Impella vs PA } \\
\text { cannula }\end{array}$ & $\begin{array}{l}\text { ECMOmax } \\
\text { (mean } \pm S D)\end{array}$ & $\begin{array}{l}\text { p-value } \\
\text { Impella vs } \\
\text { PA cannula }\end{array}$ & $\begin{array}{l}\text { ECMOmax } \\
+ \text { Vent } \\
\text { (mean } \pm S D)\end{array}$ & $\begin{array}{l}\text { p-value } \\
\text { Impella } \\
\text { vs PA } \\
\text { cannula }\end{array}$ & $\begin{array}{l}\text { p-value } \\
\text { ECMO vs } \\
\text { ECMO + } \\
\text { vent }\end{array}$ \\
\hline \multirow{2}{*}{$\begin{array}{l}\text { MAP } \\
(\mathrm{mmHg})\end{array}$} & Impella & $78.0 \pm 13.0$ & 0.298 & $54.8 \pm 8.4$ & 0.951 & $74.9 \pm 15.7$ & 0.775 & $71.6 \pm 11.8$ & 0.603 & 0.680 \\
\hline & $\begin{array}{l}\text { PA } \\
\text { cannula }\end{array}$ & $85.0 \pm 8.4$ & & $54.4 \pm 13.0$ & & $78.2 \pm 22.6$ & & $75.8 \pm 15.1$ & & 0.843 \\
\hline \multirow{2}{*}{$\begin{array}{l}\mathrm{HR} \\
\text { (bpm) }\end{array}$} & Impella & $96.0 \pm 41.1$ & 0.856 & $105.7 \pm 39.2$ & 0.598 & $99.3 \pm 36.8$ & 0.565 & $97.0 \pm 31.8$ & 0.669 & 0.908 \\
\hline & $\begin{array}{l}\text { PA } \\
\text { cannula }\end{array}$ & $92.5 \pm 23.4$ & & $95.5 \pm 26.7$ & & $89.8 \pm 16.9$ & & $89.9 \pm 25.5$ & & 0.994 \\
\hline \multirow{2}{*}{$\begin{array}{l}\mathrm{SV}_{\mathrm{c}} \mathrm{O}_{2} \\
(\%)\end{array}$} & Impella & $77.5 \pm 5.7$ & 0.870 & $66.8 \pm 7.2$ & 1.000 & $85.2 \pm 4.6$ & 0.325 & $87.0 \pm 5.0$ & 0.209 & 0.552 \\
\hline & $\begin{array}{l}\text { PA } \\
\text { cannula }\end{array}$ & $76.8 \pm 8.8$ & & $66.8 \pm 14.6$ & & $78.2 \pm 15.3$ & & $81.8 \pm 8.1$ & & 0.610 \\
\hline \multirow{2}{*}{$\begin{array}{l}\mathrm{SV}_{\mathrm{m}} \mathrm{O}_{2} \\
(\%)\end{array}$} & Impella & $67.8 \pm 6.4$ & 1.000 & $65.4 \pm 17.8$ & 0.309 & $86.0 \pm 5.8$ & 0.513 & $87.8 \pm 5.8$ & 0.342 & 0.623 \\
\hline & $\begin{array}{l}\text { PA } \\
\text { cannula }\end{array}$ & $67.8 \pm 10.1$ & & $51.8 \pm 24.3$ & & $82.5 \pm 10.7$ & & $82.5 \pm 11.3$ & & 1.000 \\
\hline \multirow{2}{*}{$\begin{array}{l}\text { PCWP } \\
\text { (mmHg) }\end{array}$} & Impella & $6.3 \pm 1.9$ & 0.969 & $6.2 \pm 4.4$ & 0.556 & $6.3 \pm 3.1$ & 0.854 & $4.6 \pm 2.5$ & 0.649 & 0.390 \\
\hline & $\begin{array}{l}\text { PA } \\
\text { cannula }\end{array}$ & $6.2 \pm 4.1$ & & $7.8 \pm 1.7$ & & $6.7 \pm 2.4$ & & $5.3 \pm 0.9$ & & 0.339 \\
\hline \multirow{2}{*}{$\begin{array}{l}\mathrm{mPAP} \\
(\mathrm{mmHg})\end{array}$} & Impella & $19.2 \pm 3.1$ & 0.228 & $31.1 \pm 6.8$ & 0.507 & $23.6 \pm 6.4$ & 0.894 & $22.0 \pm 6.9$ & 0.005 & 0.677 \\
\hline & $\begin{array}{l}\text { PA } \\
\text { cannula }\end{array}$ & $22.8 \pm 6.7$ & & $26.6 \pm 15.2$ & & $24.3 \pm 11.2$ & & $12.4 \pm 5.0$ & & 0.015 \\
\hline \multirow{2}{*}{$\begin{array}{l}\mathrm{cCO} \\
(\mathrm{L} / \mathrm{min})\end{array}$} & Impella & $6.0 \pm 1.6$ & 0.055 & $3.3 \pm 0.8$ & 0.626 & $4.0 \pm 1.0$ & 0.069 & $3.3 \pm 0.8$ & 0.568 & 0.270 \\
\hline & $\begin{array}{l}\text { PA } \\
\text { cannula }\end{array}$ & $4.4 \pm 1.0$ & & $3.0 \pm 1.0$ & & $2.8 \pm 1.0$ & & $2.9 \pm 1.2$ & & 0.886 \\
\hline
\end{tabular}

\section{UNLOADING}

PV data are summarized in Table 3. After V-A ECLS implant and start, LVEDV, SW and PVA increased. (Table 3).

Impella resulted in an immediate drop in LVEDV compared to a slight decrease in the PA cannula group, resulting in an overall SW and PVA reductions with both techniques (graphs 1 and 2). However, SW reduction was significant only in the Impella group (V-A ECLS 3998.8 $\pm 2027.6 \mathrm{mmHg} \times \mathrm{mL}$ vs V-A ECLS + Impella $1796.9 \pm 1033.9 \mathrm{mmHg} \times \mathrm{ml}, \mathrm{p}$ value=0,015), leading to a more consistent PVA reduction (Impella reduction $34,7 \%$ vs PA cannula reduction $9,7 \%$,). Furthermore, potential energy (PE) decreased by $14,8 \%$ with Impella support, whereas the PA cannula effect was negligible, showing an increasing trend (PE percentage reduction -1,2\%). Two representative PV loops regarding Impella and PA cannula groups are shown in figure 1 and figure 2. 
Table 3

LV PV variables at different time points.

\begin{tabular}{|c|c|c|c|c|c|c|c|c|c|c|}
\hline & & $\begin{array}{l}\text { Baseline } \\
\text { (mean } \pm S D)\end{array}$ & $\begin{array}{l}\text { p-value } \\
\text { Impella } \\
\text { vs PA } \\
\text { cannula }\end{array}$ & $\begin{array}{l}\text { CS } \\
\text { (mean } \pm S D)\end{array}$ & $\begin{array}{l}\text { p-value } \\
\text { Impella } \\
\text { vs PA } \\
\text { cannula }\end{array}$ & $\begin{array}{l}\text { ECMO max } \\
(\text { mean } \pm S D)\end{array}$ & $\begin{array}{l}\text { p-value } \\
\text { Impella } \\
\text { vs PA } \\
\text { cannula }\end{array}$ & $\begin{array}{l}\text { ECMOmax + } \\
\text { Vent } \\
\text { (mean } \pm S D \text { ) }\end{array}$ & $\begin{array}{l}\text { p-value } \\
\text { Impella } \\
\text { vs PA } \\
\text { cannula }\end{array}$ & $\begin{array}{l}\text { p- } \\
\text { value } \\
\text { ECMO } \\
\text { vs } \\
\text { ECMO } \\
+ \text { vent }\end{array}$ \\
\hline \multirow[t]{2}{*}{ LVEDV (ml) } & Impella & $137.2 \pm 61.8$ & \multirow[t]{2}{*}{0.198} & $118.9 \pm 75.0$ & \multirow[t]{2}{*}{0.607} & $143.6 \pm 67.4$ & \multirow[t]{2}{*}{0.766} & $123.9 \pm 75.7$ & \multirow[t]{2}{*}{0.855} & 0.633 \\
\hline & $\begin{array}{l}\text { PA } \\
\text { cannula }\end{array}$ & $98.2 \pm 42.4$ & & $101.7 \pm 33.5$ & & $134.1 \pm 39.9$ & & $130.1 \pm 34.7$ & & 0.853 \\
\hline \multirow[t]{2}{*}{ LVESV (ml) } & Impella & $68.3 \pm 58.6$ & \multirow[t]{2}{*}{0.280} & $78.9 \pm 71.7$ & \multirow[t]{2}{*}{0.664} & $96.3 \pm 64.9$ & \multirow[t]{2}{*}{0.997} & $91.4 \pm 64.1$ & \multirow[t]{2}{*}{0.730} & 0.895 \\
\hline & $\begin{array}{l}\text { PA } \\
\text { cannula }\end{array}$ & $40.8 \pm 22.4$ & & $65.4 \pm 25.6$ & & $96.4 \pm 34.0$ & & $101.1 \pm 25.0$ & & 0.785 \\
\hline \multirow{2}{*}{$\begin{array}{l}\text { LVEDP } \\
(\mathrm{mmHg})\end{array}$} & Impella & $10.9 \pm 4.8$ & \multirow[t]{2}{*}{0.479} & $9.6 \pm 4.7$ & \multirow[t]{2}{*}{0.107} & $13.3 \pm 8.5$ & \multirow[t]{2}{*}{0.927} & $7.9 \pm 6.3$ & \multirow[t]{2}{*}{0.143} & 0.250 \\
\hline & $\begin{array}{l}\text { PA } \\
\text { cannula }\end{array}$ & $8.6 \pm 5.5$ & & $20.7 \pm 14.9$ & & $12.9 \pm 4.7$ & & $13.4 \pm 5.7$ & & 0.880 \\
\hline \multirow{2}{*}{$\begin{array}{l}\text { LVESP } \\
(\mathrm{mmHg})\end{array}$} & Impella & $90.5 \pm 18.7$ & \multirow[t]{2}{*}{0.758} & $82.1 \pm 22.0$ & \multirow[t]{2}{*}{0.081} & $87.5 \pm 15.9$ & \multirow[t]{2}{*}{0.462} & $73.5 \pm 16.9$ & \multirow[t]{2}{*}{0.884} & 0.135 \\
\hline & $\begin{array}{l}\text { PA } \\
\text { cannula }\end{array}$ & $87.4 \pm 16.1$ & & $62.9 \pm 16.2$ & & $79.3 \pm 22.3$ & & $74.9 \pm 16.3$ & & 0.696 \\
\hline \multirow{2}{*}{$\begin{array}{l}\text { PVA } \\
(\mathrm{mmHg} x \\
\mathrm{mL})\end{array}$} & Impella & $8615.8 \pm 3492.0$ & \multirow[t]{2}{*}{0.241} & $5510.6 \pm 3254.3$ & \multirow[t]{2}{*}{0.310} & $8092.3 \pm 3805.1$ & \multirow[t]{2}{*}{0.177} & $5283.7 \pm 3061.8$ & \multirow[t]{2}{*}{0.941} & 0.154 \\
\hline & $\begin{array}{l}\text { PA } \\
\text { cannula }\end{array}$ & $6459.6 \pm 2904.0$ & & $3877.7 \pm 2271.0$ & & $5730.4 \pm 2071.5$ & & $5177.2 \pm 1702.9$ & & 0.613 \\
\hline \multirow{2}{*}{$\begin{array}{l}\mathrm{SW}(\mathrm{mmHg} \\
x \mathrm{~mL})\end{array}$} & Impella & $5364.7 \pm 1529.7$ & \multirow[t]{2}{*}{0.473} & $2407.2 \pm 776.3$ & \multirow[t]{2}{*}{0.346} & $3998.8 \pm 2027.6$ & \multirow[t]{2}{*}{0.026} & $1796.9 \pm 1033.9$ & \multirow[t]{2}{*}{0.457} & 0.016 \\
\hline & $\begin{array}{l}\text { PA } \\
\text { cannula }\end{array}$ & $4624.3 \pm 2022.2$ & & $1792.0 \pm 1407.0$ & & $1961.9 \pm 1054.0$ & & $1361.7 \pm 1000.8$ & & 0.309 \\
\hline \multirow{2}{*}{$\begin{array}{l}\mathrm{PE}(\mathrm{mmHg} \\
x \mathrm{~mL})\end{array}$} & Impella & $3251.2 \pm 2792.3$ & \multirow[t]{2}{*}{0.245} & $3103.5 \pm 3076.8$ & \multirow[t]{2}{*}{0.444} & $4093.5 \pm 2784.9$ & \multirow[t]{2}{*}{0.798} & $3486.8 \pm 2868.3$ & 0.797 & 0.710 \\
\hline & $\begin{array}{l}\text { PA } \\
\text { cannula }\end{array}$ & $1835.2 \pm 1108.3$ & & $2085.7 \pm 1106.9$ & & $3768.5 \pm 1379.7$ & & $3815.5 \pm 1243.8$ & & 0.951 \\
\hline $\mathrm{E}_{\mathrm{ES}}$ & Impella & $3.3 \pm 3.1$ & 0.602 & $2.1 \pm 2.1$ & 0.406 & $1.4 \pm 0.9$ & 0.210 & $1.2 \pm 0.7$ & 0.174 & 0.667 \\
\hline & $\begin{array}{l}\text { PA } \\
\text { cannula }\end{array}$ & $2.6 \pm 1.1$ & & $1.3 \pm 1.1$ & & $0.9 \pm 0.4$ & & $0.8 \pm 0.2$ & & 0.583 \\
\hline Tau (ms) & Impella & $31.0 \pm 9.3$ & 0.212 & $45.1 \pm 20.6$ & 0.763 & $50.9 \pm 16.1$ & 0.400 & $50.0 \pm 12.4$ & 0.231 & 0.914 \\
\hline & $\begin{array}{l}\text { PA } \\
\text { cannula }\end{array}$ & $37.5 \pm 8.9$ & & $48.3 \pm 12.7$ & & $57.4 \pm 10.1$ & & $58.2 \pm 10.0$ & & 0.895 \\
\hline
\end{tabular}

\section{Discussion}

The use of Impella or PA cannula, in association with V-A ECLS, were able to unload the LV in a large closed-chest porcine model of profound CS. However, Impella provided more effective LV unloading than PA cannula, whereas no marked difference between the two V-A ECLS configurations was observed with regards to end organ perfusion.

\section{Effect of Peripheral V-A ECLS on LV Unloading}

Our study confirmed what has been repetitively demonstrated (Burkhoff, Donker, etc....) that V-A ECLS in CS is associated with PVA increase ${ }^{12} 13$. Indeed, V-A ECLS leads to LV afterload increase, thereby moving the Ea line to the right (if we consider TPR and LV contractility as constants). In this condition, overcoming the afterload is only achievable via the Starling 's Law, that is rising LVEDV ${ }^{14}$. As a consequence, LVEDP, LA pressure, and PCWP increase. The global effect is the shifting of PV loop rightward and upward along the EDPVR, becoming progressively narrower and taller. Translating these changes into hemodynamic terms: PVA increases, rising subendocardial pressure, and increasing myocardial oxygen demand ${ }^{15}$, all negative factors for an injured myocardium. In the extreme circumstances, these adverse events are such that unloading the LV during V-A ECLS support becomes mandatory. 
Our study showed that both direct LV unloading with Impella and indirect LV unloading with PA drainage in combination of right-sided V-A ECLS unloading, led to more effective decompression.

\section{Impella}

The Impella decompress LV by pumping continuously blood from the LV to the aorta. Losing the isovolumetric periods, PV loops attains a triangular shape and shifts gradually leftwards, according to the flow generated by Impella ${ }^{8}$ (figure1). In this animal series, we observed a decremental trend in LVEDV, LVEDP and slightly in PCWP. These findings are consistent with the effects on the pulmonary and circulatory physiology founded in other animal studies ${ }^{16} 1718$. In addition, Lim et al confirmed this hemodynamic impact in a single-center study of six consecutive patients on V-A ECLS support who received LV unloading with Impella ${ }^{19}$.

The current study showed how Impella is able to impact effectively on PCWP rather than on mean PAP, therefore on the overall pulmonary circulation. On one hand, Yourshaw et al showed how the maximum Impella effect on PCWP was recorded 12 hours later from the device implantation ${ }^{20}$. This may lead to consider a slow hemodynamic adaptation which might not be seen in the short data capture interval used in our protocol. On the other hand, the PCWP is the left side parameter which might theoretically be more influenced by percutaneous left ventricle assistance.

To summarize, our findings showed how Impella had major effects on PCWP and LVEDV. This was found also in a simulation study performed by Donker and collaborators in a closed-loop real-time computer model of $\mathrm{CS}^{13}$.

\section{Pulmonary Artery Drainage}

PA drainage with percutaneous cannulation use has been recently reported in the literature ${ }^{21} 22$. Scanty data, however, are available regarding its actual hemodynamic impact in V-A ECLS. Von Segesser et al. first reported the potential advantages of pulmonary cannulation in five bovine model of CS. The placement of a pulmonary arterial cannula allowed LV decompression. In fact, mean PAP sharply dropped. This latter hemodynamic change led to lower LV pressure and higher aortic pressure ${ }^{23}$. Our experience confirmed these findings, since mean PAP dramatically decreased by approximately fifty percent as soon as PA cannula was placed. However, this action seems to have a limited impact in terms of LV volume reduction, as suggested by the slight LVEDV decrease.

\section{UNLOADING EFFECTIVENESS}

Our study aimed at analyzing two techniques for unloading the LV in combination of V-A ECLS, considering PVA as a satisfactory LV overload estimator. PVA linearly correlates with myocardial oxygen consumption, independently from the heart rate ${ }^{24}$. Impella and PA cannula were able to reduce PVA in this animal model of CS and, therefore, both strategies represent effective means to unload the LV during peripheral V-A ECLS, in case of need.

PVA is the total mechanical energy performed by the ventricle per heartbeat, which is the sum of SW (external active forces) and potential energy (PE, internal passive forces) ${ }^{8}$.

In terms of external forces, SW significantly dropped by more than fifty percent with Impella, whereas this reduction was 30,6\% in PA cannula group.

Furthermore, the impact of PA cannula and Impella on internal passive energy was extremely different. While PA cannula showed to have not consistent impact on the potential energy of the LV, PE dropped remarkably in the Impella group.

Therefore, take into account the fact that PVA reduction is more than three times higher with Impella, the latter technique seems to be more effective in terms of unloading. This means being able to reduce both PVA components - either internal or external energy. Drainage through the PA cannula, instead, seems to influence only the external force generated by the LV.

\section{END-ORGAN PERFUSION}

A satisfactory treatment of CS means interrupting the vicious cycle of myocardial dysfunction leading to low cardiac output and hypotension ${ }^{25}$, and restoring end-organ perfusion ${ }^{26}$. In our study, mixed and central venous oxygen saturation were considered adequate surrogates of organ perfusion ${ }^{27}$.

Indeed, mean arterial pressure and venous oxygen saturation clearly improved just with the institution of V-A ECLS support. This improvement occurred at the expense of LV loading due to higher LVEDV, LVEDP and PVA and thereby increased myocardial oxygen consumption secondary to the higher afterload generated by the retrograde ECLS-related flow. The start of the two unloading techniques, end-organ perfusion parameters (as $\mathrm{MAP}$ and $\mathrm{SVcO} \mathrm{O}_{2}$ or $\mathrm{SVmO}_{2}$ ) were successfully kept stable in association with LV unloading. PVA and consequently myocardial oxygen consumption dropped with both techniques, which might protect the myocardium against further ischaemic injury or enhance, and make more expeditious, the myocardial recovery during ECLS support ${ }^{28}$.

Furthermore, the fact that five out of six animals supported with PA cannula required norepinephrine to maintain a MAP $>50$ mmHg is probably due to the adjunctive contribution of the Impella to the circulatory support in association with V-A ECLS assistance, contribution which it is absent in case of PA drainage.

\section{Limitations}

First, this is a study designed on acute immediate term effects of the Impella and PA cannula on top of V-A ECLS and it is unknown if the loading effects would become greater with long-term monitoring. Secondly, venous oxygen saturations were the only biochemical parameters of end-organ perfusion. The

Page 7/10 
lack of lactates in our experience might represent a consider weakness when perfusion was evaluated. Third, pigs have a short ascending aorta, thereby the Impella outflow might be placed in the aortic arch/descending aorta, possibly jeopardizing the device impact understanding. Another limitation to our study is the impossibility to assess the LV unloading strategy effects at a longer-term outcomes, as well as defining infarct size, as the microcirculation is obstructed. Fourth, the gold standard for estimating ESPVR and PVA is by preload reduction which was not applicable in this study as we mentioned in methods section. Further, $\mathrm{V}_{0}$ was arbitrarily set as $0 \mathrm{ml}$ in all animals and therefore might influence subsequently the absolute values of the relative derived variables.

Finally, Impella and PA cannula are only two among several LV venting techniques nowadays available ${ }^{8}$. Little is known how their different combinations with V-A ECLS affect the LV and end-organ perfusion, indicating the need for further studies.

\section{Conclusions}

This is the first study which directly compared the effects of two different modalities (one direct and one indirect) of the LV during peripheral V-A ECLS. In this large animal model with profound CS due to complete balloon-based proximal/middle left anterior descendent occlusion, the Impella and PA cannula, in association with V-A ECLS, provided effective LV unloading maintaining adequate end-organ perfusion. Impella seems to guarantee a stronger LV unloading effect, reducing more effectively the total LV mechanical energy.

\section{Declarations}

Ethics approval and consent to participate

The study was approved by Charles University, First Faculty of Medicine Institutional Animal Care and Use Committee and was performed at the university laboratory in accordance with Act No 246/1992 Coll. on the protection of animals against cruelty that is harmonized with EU directives on the protection of animals used for scientific purposes.

Consent for publication

Not applicable

Availability of data and materials

The datasets used and/or analysed during the current study are available from the corresponding author on reasonable request.

Competing interests

The authors declare that they have no competing interests

Funding

This work was supported by Euro ELSO research grant 2017 and by the Department for the Treatment and Study of Cardiothoracic Diseases and Cardiothoracic Transplantation, Mediterranean Institute for Transplantation and Advanced Specialized Therapies (ISMETT, Palermo, Italy)

Authors' contributions

All authors contributed to the design and implementation of the research, to the analysis of the results and to the writing of the manuscript.

Acknowledgements

Not applicable

\section{References}

1 Hochman JS, Sleeper LA, Webb JG Early revascularization and long-term survival in cardiogenic shock complicating acute myocardial infarction. JAMA. 2006 Jun 7;295(21):2511-5.

2 Thiele H, Akin I, Sandri M et al. One-Year Outcomes after PCI Strategies in Cardiogenic Shock.N Engl J Med. 2018 Nov 1;379(18):1699-1710. doi:

10.1056/NEJMoa1808788. Epub 2018 Aug 25.

3 Vahdatpour C., Collins D., Goldberg S. Cardiogenic Shock. J Am Heart Assoc. 2019 Apr 16;8(8):e011991.

4 Meani P, Gelsomino S, Natour E, et al. Modalities and effects of left ventricle unloading on extracorporeal life support: a review of the current literature. Eur J Heart Fail 2017; 19: 84-91.

5 Ostadal P, Mlcek M, Kruger A, et al. Increasing venoarterial extracorporeal membrane oxygenation flow negatively affects left ventricular performance in a porcine model of cardiogenic shock. J Transl Med 2015;13:266.

6 Pappalardo F, Schulte C, Pieri M, et al. Concomitant implantation of Impella ${ }^{\circledR}$ on top of veno-arterial extracorporeal membrane oxygenation may improve survival of patients with cardiogenic shock. Eur J Heart Fail. 2017;19:404-12.

Page $8 / 10$ 
7 Kawashima D, Gojo S, Nishimura T, Itoda Y, Kitahori K, Motomura N, et al. Left ventricular mechanical support with Impella provides more ventricular unloading in heart failure than extracorporeal membrane oxygenation. ASAIO journal (American Society for Artificial Internal Organs : 1992). 2011;57(3):16976.

8 Jensen PB, Kann SH, Veien KT, et al. Single-centre experience with the Impella CP, 5.0 and RP in 109 consecutive patients with profound cardiogenic shock Eur Heart J Acute Cardiovasc Care, 7 (1) (2018), pp. 53-61

9 Tepper S, Masood MF, Baltazar Garcia M, et al. Left Ventricular Unloading by Impella Device Versus Surgical Vent During Extracorporeal Life Support. Ann Thorac Surg 2017;104:861-7.

10 Sagawa K. The end-systolic pressure-volume relation of the ventricle: definition, modifications and clinical use. Circulation. 1981;63:1223-7.

11 Senzaki H, Chen $\mathrm{CH}$, Kass DA. Single-beat estimation of end-systolic pressure-volume relation in humans. A new method with the potential for noninvasive application. Circulation. 1996;94: 2497-506.

12 Bastos M, Burkhoff D, Maly J, et al. Invasive left ventricle pressure-volume analysis: overview and practical clinical implications. Eur Heart J. 2019;ehz552

13 Donker DW, Brodie D, Henriques JPS, Broomé M. Left ventricular unloading during veno-arterial ECMO: a simulation study. ASAIO J 2018. Mar 10.

14 Burkhoff D, Sayer G, Doshi D, Uriel N. Hemodynamics of Mechanical Circulatory Support. Journal of the American College of Cardiology. 2015;66(23):2663-74.

15 Soleimani B, Pae WE. Management of left ventricular distension during peripheral extracorporeal membrane oxygenation for cardiogenic shock. Perfusion. 2012;27(4):326-31.

16 Saku K, Kakino T, Arimura T, et al. Left Ventricular Mechanical Unloading by Total Support of Impella in Myocardial Infarction Reduces Infarct Size, Preserves Left Ventricular Function, and Prevents Subsequent Heart Failure in Dogs. Circ Heart Fail. 2018;11(5):e004397.

17 Watanabe S, Fish K, Kovacic JC, et al. Left Ventricular Unloading Using an Impella CP Improves Coronary Flow and Infarct Zone Perfusion in Ischemic Heart Failure. J Am Heart Assoc. 2018;7(6):e006462

18 Weil BR, Konecny F, Suzuki G, lyer V, Canty JM Jr. Comparative Hemodynamic Effects of Contemporary Percutaneous Mechanical Circulatory Support Devices in a Porcine Model of Acute Myocardial Infarction. JACC Cardiovasc Interv. 2016;9(22):2292-2303.

19 Lim HS. The Effect of Impella CP on Cardiopulmonary Physiology During Venoarterial Extracorporeal Membrane Oxygenation Support. Artificial organs. 2017.

20 Yourshaw JP, Mishra P, Armstrong MC, et al. Effects of Percutaneous LVAD Support on Right Ventricular Load and Adaptation. J Cardiovasc Transl Res. 2019;12(2):142-149.

21 Avalli L, Maggioni E, Sangalli F, Favini G, Formica F, Fumagalli R: Percutaneous left-heart decompression during extracorporeal membrane oxygenation: an alternative to surgical and tran- septal venting in adult patients. ASAIO J 57: 38-40, 2011.

22 Loforte A, Baiocchi M, Dal Checco E, Percutaneous Pulmonary Artery Venting via Jugular Vein While on Peripheral Extracorporeal Life Support.ASAIO J. 2019 Mar 19.

23 von Segesser LK, Kwang K, Tozzi P, Horisberger J, Dembitsky W: A simple way to decompress the left ventricle during venoarterial bypass. Thorac Cardiovasc Surg 56: 337-341, 2008

24 Suga H. Ventricular energetics. Physiol Rev. 1990;70:247-77

25 Reynolds HR, Hochman JS. Cardiogenic shock: current con- cepts and improving outcomes. Circulation. 2008;117:686-97.

26 Haas SA, Lange T, Saugel B, Petzoldt M, Fuhrmann V, Metschke M, Kluge S. Severe hyperlactatemia, lactate clearance and mortality in unselected critically ill patients. Intensive Care Med. 2016;42:202-10.

27 Frank Bloos Konrad Reinhart Venous oximetry Intensive Care Medicine July 2005, Volume 31, Issue 7, pp 911-913

28 Maroko PR, Kjekshus JK, Sobel BE, Watanabe T, Covell JW, Ross J Jr, Braunwald E. Factors influencing infarct size following experimental coronary artery occlusions. Circulation. 1971;43: 67-82

\section{Graphs}

Due to technical limitations, the graphs are available as a download in the supplemental files section.

Graph 1. Pressure Volume Energy Changes in the PA cannula Group at different time points.

Page $9 / 10$ 


\section{Figures}
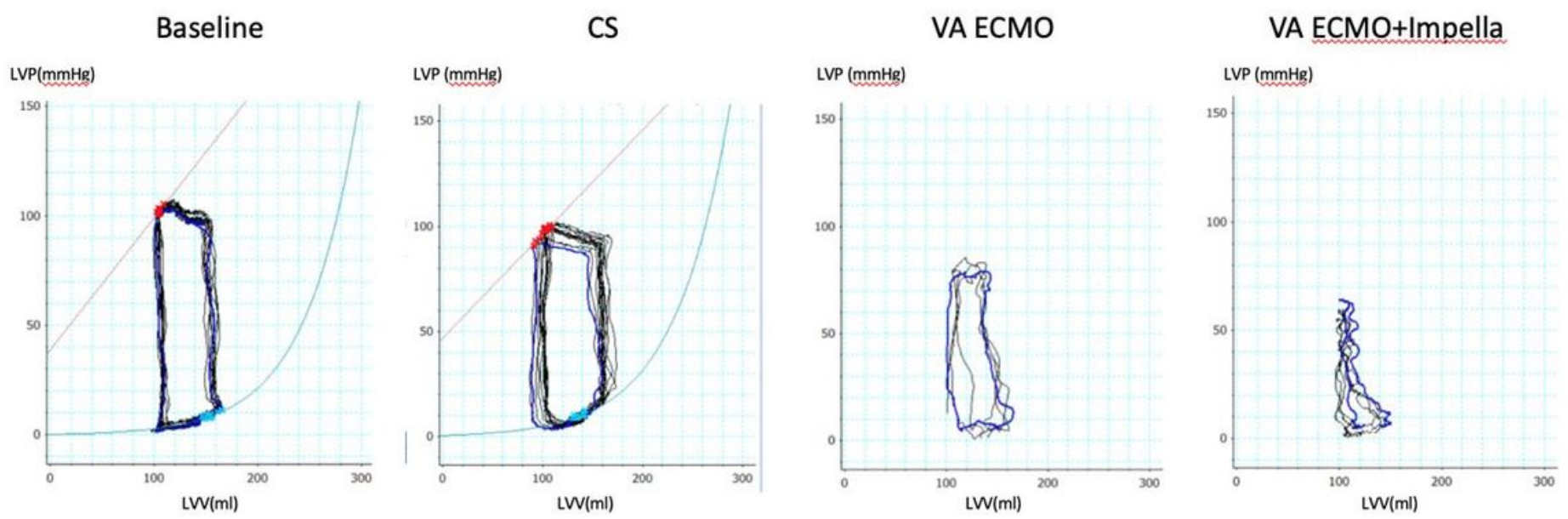

Figure 1

Representative Animal: PV loop CS VA ECMO + Impella

Baseline

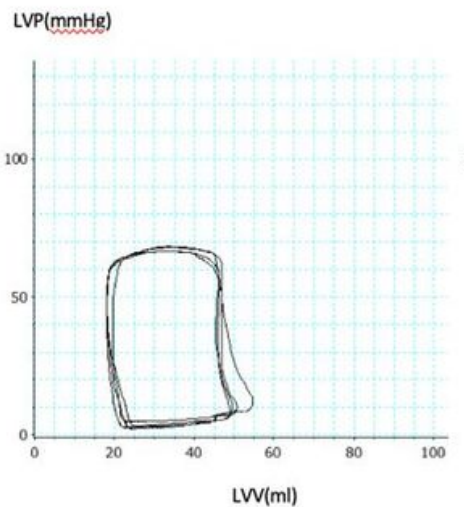

CS

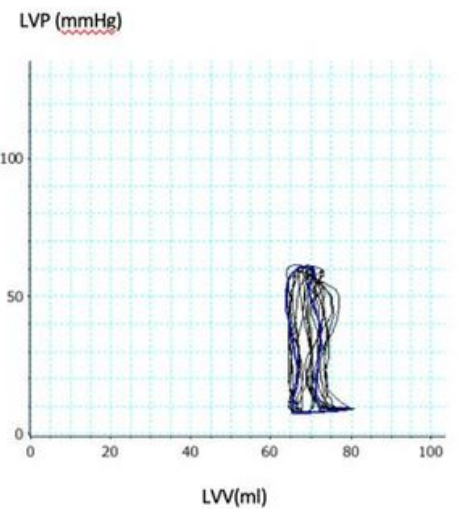

VA ECMO

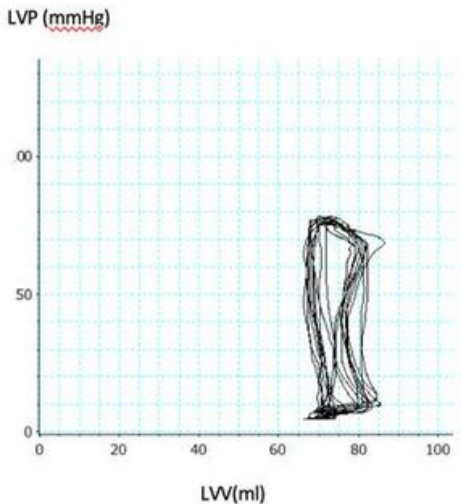

\section{VA ECMO+PA cannula}

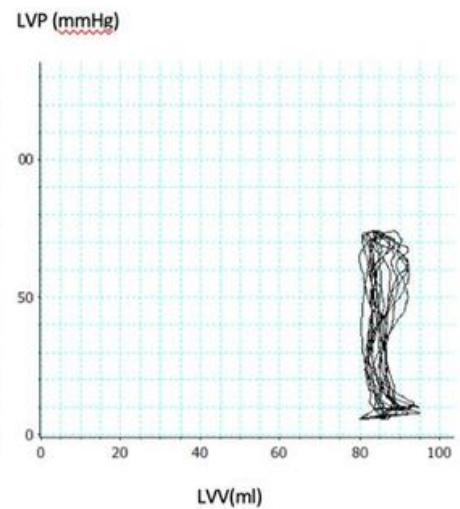

Figure 2

Representative Animal:PV loop CS VA ECMO + PA cannula

\section{Supplementary Files}

This is a list of supplementary files associated with this preprint. Click to download.

- Appendix260220.docx

- Graph2.JPG

- Graph1.JPG 